\title{
A new periodic interdigital structure - three dimensional realization based on two dimensional design
}

\author{
H. Ning \\ ninghuansheng@buaa.edu.cn \\ J. Wang \\ Q. Xiong \\ L. Mao
}

\author{
School of Electronic and Information Engineering, Beihang University, Beijing 100191, China \\ School of Electronic and Information Engineering, Beihang University, Beijing 100191, China \\ School of Electronic and Information Engineering, Beihang University, Beijing 100191, China
}

Institute of Intelligent Structure and System, Soochow University, Suzhou 215006, China

A novel design concept of new periodic interdigital structure for total transmission is proposed from the perspective of electromagnetic (EM) field in this paper. The new structure is constructed in two-dimensional (2D) firstly by using the constructive interference of EM fields, and its transmission characteristic and band structure are calculated. Based on the calculated results, the interaction of the structure with EM fields is clearly demonstrated to provide a better physical understanding for the structure design. Meanwhile, the final three-dimensional (3D) implementation of the presented structure in actual printed circuit board is conducted for its potential applications. The maximum transmission frequency, bandgap and electric field distribution calculated by using the $2 \mathrm{D}$ structure and its final implementation all testify the validity of the realization process.

[DOI: http://dx.doi.org/10.2971/jeos.2012.12030]

Keywords: periodic structure, photonic bandgap, electromagnetic bandgap

\section{INTRODUCTION}

Periodic structures composed of dielectric, metal or metallodielectric element exhibit a bandgap prohibiting the propagation of electromagnetic (EM) fields [1]. When periodic structures are operated at microwave and millimeter-wave frequencies, they are often referred as electromagnetic bandgap (EBG) structures. Recently, EBG structures have attracted much attention due to their wide applications in developing components for microwave and millimeter-wave devices as well as in antenna design [2]-[10]. For instance, EBG structures provide a popular solution to the mutual coupling reduction in patch antenna arrays [10].

Some effective methods have been developed to analyze the characteristics of EBG structures, which mainly include direct numerical simulation of the structure [2]-[5] and the equivalent circuit model [6]-[10]. However, the underlying physical understanding of the aforementioned methods needs to be further investigated. To be more specific, the interaction of the structures with EM fields needs to be further revealed to provide a more general design methodology of periodic structure. Moreover, most of the research has been focused on the bandgap of periodic structures and little has been investigated on other characteristics of these structures.

In this paper, a novel design concept of new periodic interdigital structure for total transmission is presented from the perspective of EM field to provide a solution to the aforementioned problems. Firstly, the new interdigital structure for to- tal transmission is constructed in $2 \mathrm{D}$ by using the constructive interference of EM fields. To reveal the interaction of the structure with EM fields, a complex permittivity obtained by the Drude model is set for the metal and there is no conduction current in the finite domain time difference (FDTD) calculation. The transmission response of the proposed structure is calculated to demonstrate the above design concept and the band structure is analyzed to investigate the bandgap. Then, the final 3D realization of the presented structure in practical printed circuit board (PCB) is conducted for its potential applications. A reference structure is developed as a benchmark. And the final implementation of the proposed structure and reference structure are simulated and measured to verify the realization process.

\section{NEW INTERDIGITAL STRUCTURE IN 2D}

Currently, one of the most important device developments concentrates on the structure whose electronic behavior is essentially 2D. In this paper, a metallic periodic structure for total transmission at microwave frequencies shown in Figure 1 is established in 2D from the perspective of constructive interference of EM fields. Figure 1(a) shows the unit cell of the new interdigital structure, which consists of a pair of anti-parallel interdigital located at a certain distance $W$. To verify above design concept, the transmission characteristic of the unit cell 


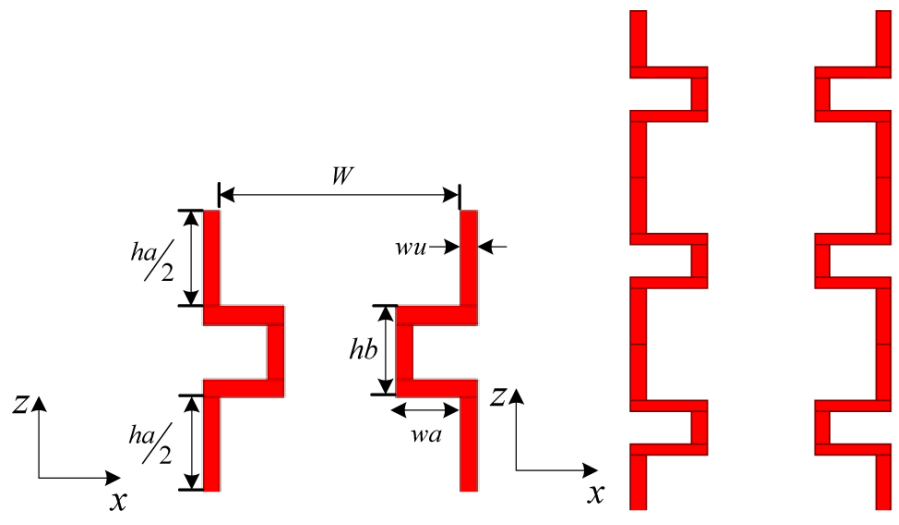

FIG. 1 Configuration of the proposed new interdigital structure in 2D. (a) Unit cell, (b) three unit cells.

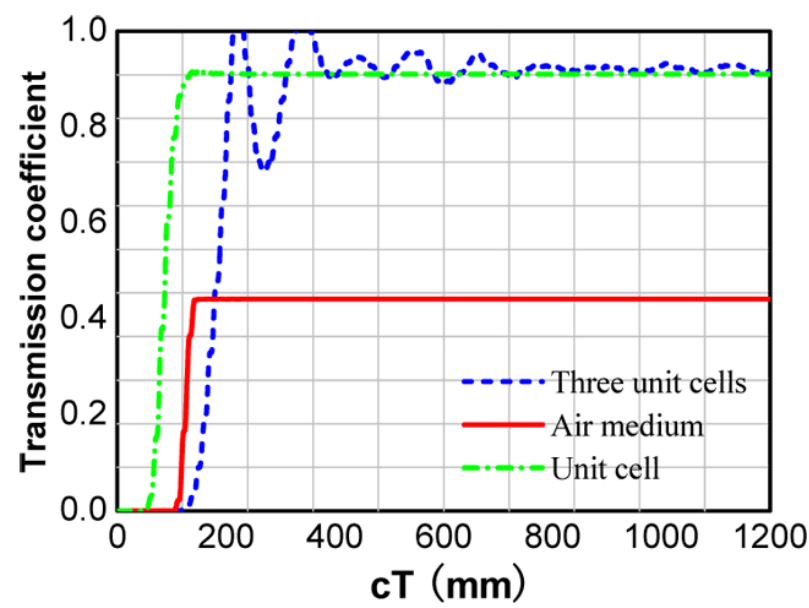

FIG. 2 Calculated transmission response when the incident wave propagates along the unit cell and three unit cells of periodic interdigital structure as well as in the air medium.

and three unit cells shown in Figure 1(b) are calculated by the commercial software RSOFT Fullwave.

In the FDTD simulations, the width of the copper $w u$ is set as $2 \mathrm{~mm}$ and other dimensions of the unit cell are chosen as: $h a=21 \mathrm{~mm}, h b=10 \mathrm{~mm}, w a=8 \mathrm{~mm}, W=30 \mathrm{~mm}$. The incident TE wave (electric field perpendicular to the plane of incidence) propagates along the $z$ direction and has a wavelength of $22 \mathrm{~mm}$. The grid size and the time step are chosen as $0.2 \mathrm{~mm}$ and 100/c s (where c is the velocity of electromagnetic wave in mm unit) respectively. Meanwhile, a $50 \mathrm{~mm}$ width of perfectly matched layer (PML) is added to both sides of the model along the $x$ direction in RSOFT Fullwave. The metal is chosen as $\mathrm{Cu}$ material and its relative permittivity is set to be $-1.596 \times 10^{5}-\mathrm{j} \times 7.65 \times 10^{7}$, which is calculated by using following Drude model [11] at $13.6 \mathrm{GHz}$.

$$
\varepsilon_{r}^{\prime}(\omega)=1-\frac{\omega_{p}^{2}}{\omega^{2}+\omega_{r}^{2}}-\mathrm{j} \frac{\omega_{p}^{2} \omega_{r}}{\omega\left(\omega_{r}^{2}+\omega^{2}\right)}
$$

with

$$
\omega_{p}^{2}=\frac{n e^{2}}{\left(m \varepsilon_{0}\right)}, \quad \omega_{r}=\frac{1}{\tau}
$$

where $n$ is conduction electron density, $e$ is the magnitude of the electron charge, $\tau$ is the relaxation time, $m$ is the electron mass, and $\varepsilon_{0}$ is the permittivity of free space.
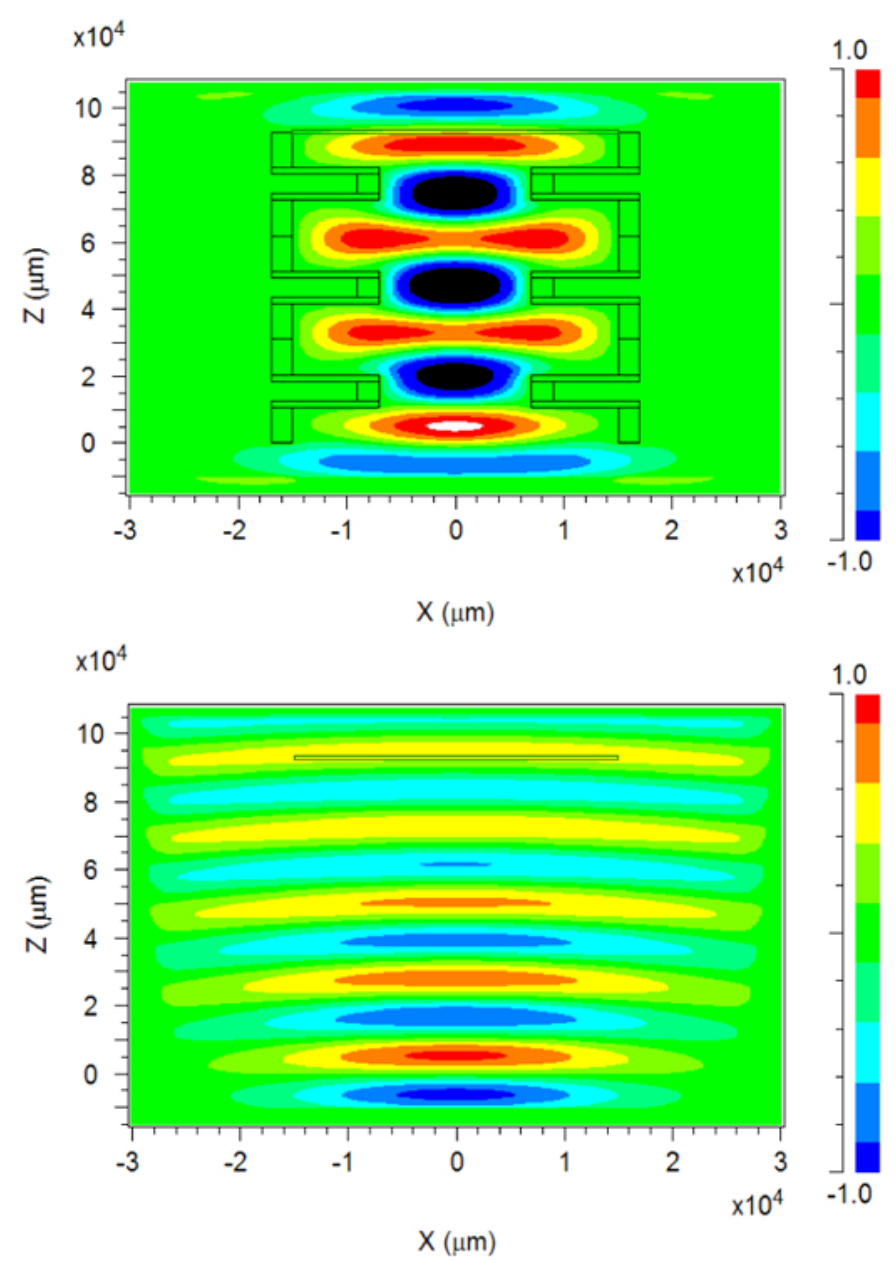

FIG. 3 Electric field distribution when the incident wave propagates along the periodic interdigital structure (a) and in the air medium (b).

To investigate the steady state output of the new interdigital structure, the time averaged response of power transmission coefficient $C_{T}(t)$ is used and calculated by following equation [12]:

$$
C_{T}(t)=\left|\frac{2}{T} \int_{t-T}^{t}\right| S(t)|d t|
$$

with

$$
S(t)=\frac{\operatorname{Re}\left[\int_{A_{1}}\left(\mathbf{E}(t) \times \mathbf{H}^{*}(t)\right) \cdot d \vec{A}_{1}\right]}{\operatorname{Re}\left[\int_{A_{2}}\left(\mathbf{E}\left(t_{0}\right) \times \mathbf{H}^{*}\left(t_{0}\right)\right) \cdot d \vec{A}_{2}\right]}
$$

where $T$ is the period of incident wave, $t_{0}$ is the initial time, $\mathbf{E}$ is electric field intensity, $\mathbf{H}$ is magnetic field intensity, $A_{1}$ and $A_{2}$ are the $\mathrm{XY}$ plane located within the calculated domain at the source and output of the structure respectively.

Through the calculation of Eq. (3), the transmission response of the proposed structure can be evaluated. Meanwhile, the transmission response under the circumstance of the incident wave propagating in the air medium is also investigated for comparison. The simulated results of transmission response are shown in Figure 2. It is observed that when the incident wave propagates along the unit cell and three unit cells of the proposed structure, the steady power transmission coefficient are $91.0 \%$ and $91.9 \%$ respectively, demonstrating the nearly total transmission characteristic of the proposed structure; while the incident wave propagates in the air medium, there is only 
$44.2 \%$ power transmission. The response time of steady output of three unit cells is longer than that of the unit cell, which is easy to understand from the viewpoint of the propagation of EM fields. By tuning the length $h a$ and $h b$ of the proposed structure, the response time can also be adjusted. Namely, the introduced phase shift by the interdigital structure may be easily controlled and this will facilitate its potential applications.

The nearly total transmission phenomenon of the new interdigital structure can be explained qualitatively by the constructive interference between the travelling fields in the proposed structure occurred at the specified frequency [13]. When the proposed structure is absent, the incident wave propagates uniformly in the infinite free space and only limited power is received within a specific range. This can be verified by the electric field (E-field) distribution shown in Figure 3 .

It is worth mentioning that in the FDTD calculations, a complex permittivity is set for the metal and there is no conduction current so that the analysis of the structure is based on EM field instead of the circuit model. Through this way, the interaction of the structure with EM fields is clearly clarified to better understand the physical meaning behind the structure design. To be specific, we can know that how the incident wave propagates along the structure and when the response is stable. Meanwhile, since the analysis and design of the structure are conducted in $2 \mathrm{D}$, the simulation time and computation memory will be greatly reduced.

The band structure of the presented structure is also calculated to investigate the bandgap and the calculation is performed by RSOFT BandSOLVE. Here, the FDTD method is used to extract frequency eigenvalues by identifying the peaks in spectrum response of the structure. The unit cell shown in Figure 1(a) is employed in the band structure calculation, which has real space lattice vector of $\left(b \hat{e}_{x}+a \hat{e}_{z}\right)$ and reciprocal lattice vector of $\left(2 \pi / b \hat{k}_{x}+2 \pi / a \hat{k}_{z}\right)$, where $a=h a+h b$ is the period along z-direction and $b=W+2 * w u+5$ is the width of calculated domain. Figure 4(a) shows the wave vectors of Brillouin zone, where $\Gamma$ is the central point, Mis located at $k_{x}=0, k_{z}=\pi / a$ and $K$ is located at $k_{x}=0, k_{z}=-\pi / a$. To obtain the variation of wave vectors along the $z$-direction, a periodic boundary condition and a PML boundary condition are applied in the $\mathrm{z}$-direction and $\mathrm{x}$-direction respectively. The calculated TE band structure is shown in Figure 4(b), where an obvious bandgap and a minor bandgap marked as yellow shaded region can be observed. Hence, the EM fields at these frequencies cannot propagate in the new interdigital structure.

\section{FINAL 3D REALIZATION}

\subsection{Considerations}

For potential applications, the final 3D realization of new interdigital structure in actual PCBs should be investigated. The periodic dielectric structure presented in $[14,15]$ is actually a 2D structure. In the concrete EBG waveguide application, the periodic structure is covered with perfect electric conduc-

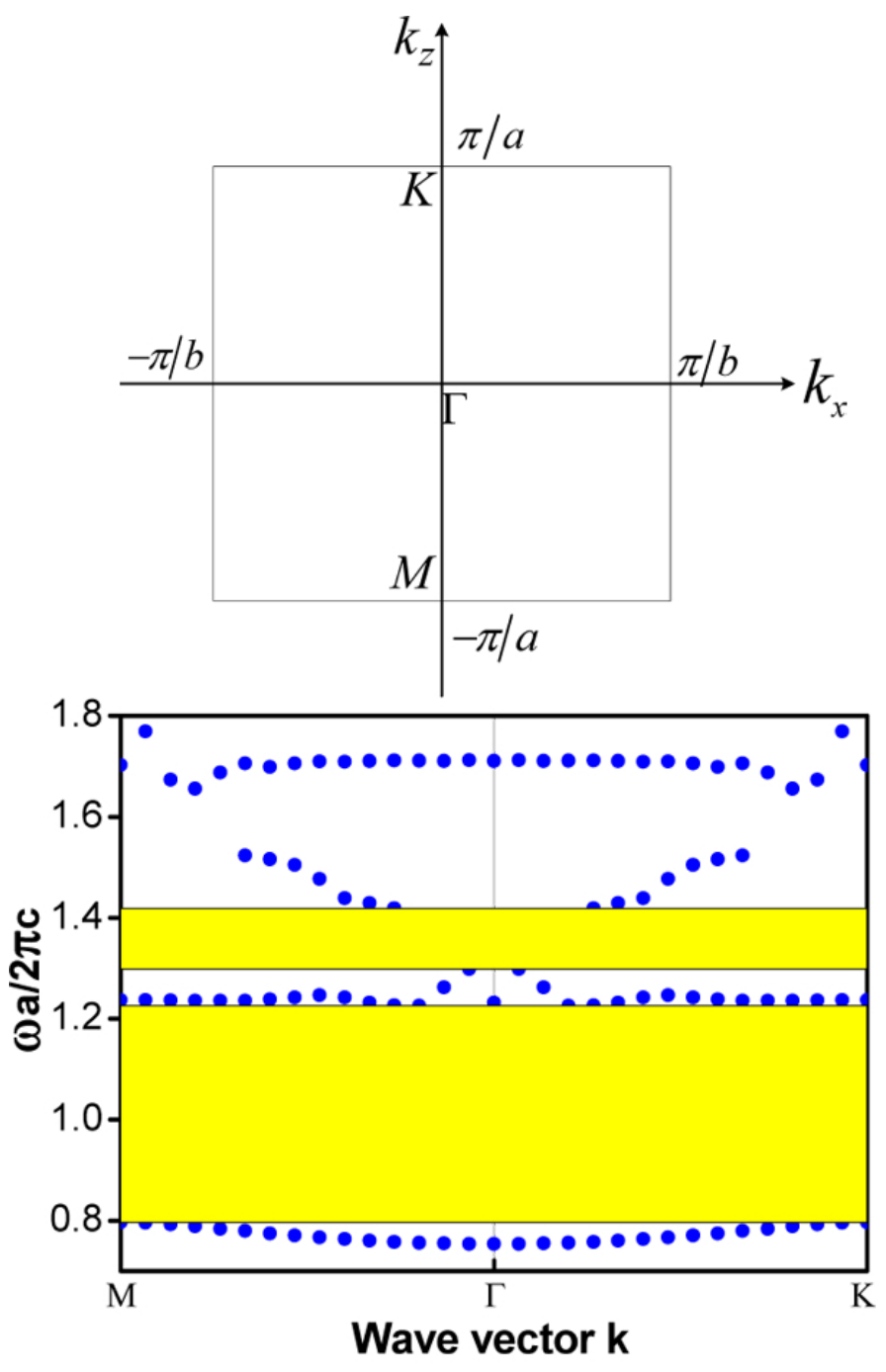

FIG. 4 (a) Brillouin zone of the proposed structure. (b) TE band structure along the z-direction.

tor (PEC). This configuration can be explained from the viewpoint of energy confinement, which means in $2 \mathrm{D}$, the incident power can be easily confined by the structure for transmission; while in the final 3D realization, the method for energy confinement needs to be accomplished to achieve the same behavior with $2 \mathrm{D}$ condition. Therefore, the top and bottom PEC are introduced.

As for the metallic structure shown in Figure 1, the incident power can be propagated through the air and constrained by the metal at both sides. For the final realization in actual PCB, we need to confine the power in the same way for transmission. Henceforth, the interdigital structure is reversed and a bottom ground plane is added. Specifically, the air medium in $2 \mathrm{D}$ corresponds to the metal of top plane in $3 \mathrm{D}$ realization and the metal in $2 \mathrm{D}$ corresponds to the air medium of top plane in $3 \mathrm{D}$ realization. The periodic structure at the top plane of final $3 \mathrm{D}$ realization can be regarded as the complementary counterpart of the interdigital structure in 2D.

The efficient excitation of the structure should be investigated as well. A microstrip line is used because of its fundamental mode where the electric field perpendicular to the substrate and the magnetic field parallel to the substrate surface [16], 


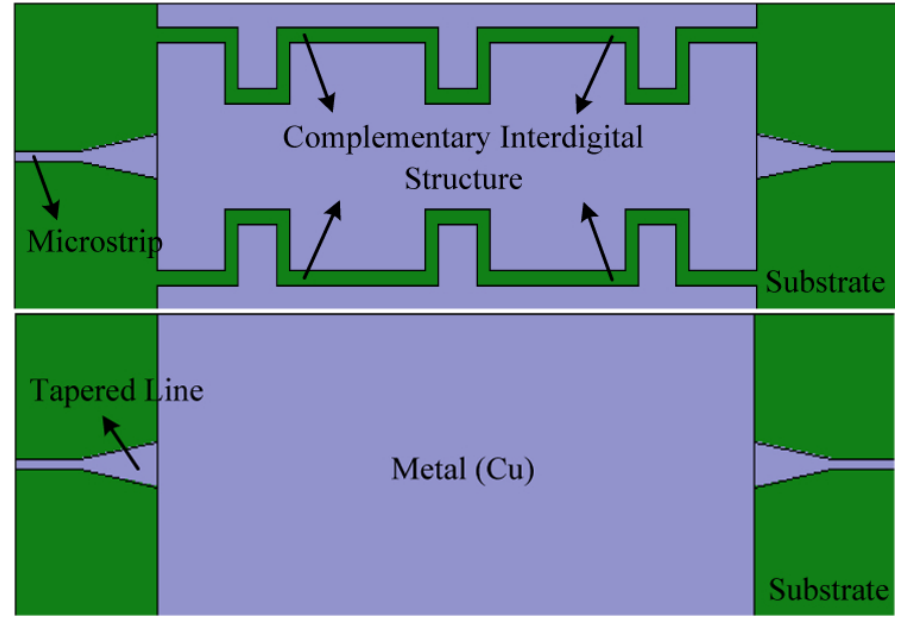

FIG. 5 (a) Top view of the final 3D realization of the proposed structure. (b) Top view of the reference structure. (The bottom ground planes are all not shown).
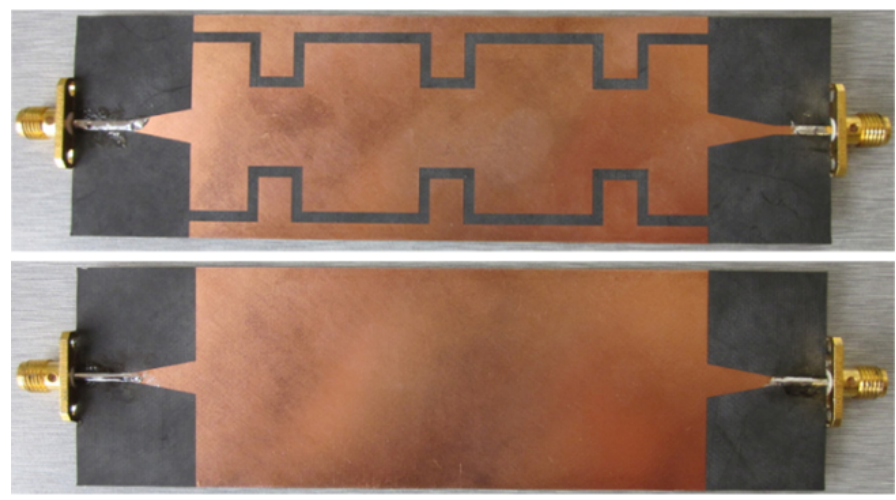

FIG. 6 Photograph of the fabricated final 3D realization of (a) the proposed structure and (b) the reference structure (the bottom ground planes are all not shown).

and this excitation is consistent with the excitation used in 2D FDTD calculation essentially. Meanwhile, a tapered transition line is utilized to reduce the reflection coefficient caused by microstrip discontinuity. The correctness of this implementation process will be testified in the following section.

\subsection{Results and discussions}

Based on above considerations, the schematic layout of final realization for the proposed structure is shown in Figure 5(a), where the middle part at the top plane of the substrate is the complement of new interdigital structure in $2 \mathrm{D}$. To evaluate the performance of proposed structure, a reference structure shown in Figure 5(b) is constructed with the same dielectric substrate, microstrip line and tapered transition. The dielectric substrate with a relative permittivity of 2.2 and thickness of $0.5 \mathrm{~mm}$ is employed in the simulation model, and the bottom ground plane of the substrate is covered with $\mathrm{Cu}$ material with conductivity of $5.8^{*} 10^{7} \mathrm{~S} / \mathrm{m}$. The width of microstrip line is $1.5 \mathrm{~mm}$ corresponding to $50 \Omega$ characteristic impedance and the length is chosen as $10 \mathrm{~mm}$. The tapered transition line with transition length of $12 \mathrm{~mm}$ and width of $6 \mathrm{~mm}$ is placed in order to excite the structure more efficiently by reducing reflection coefficient. The total width is chosen as $40 \mathrm{~mm}$.

The ultimate realizations of the proposed structure and the

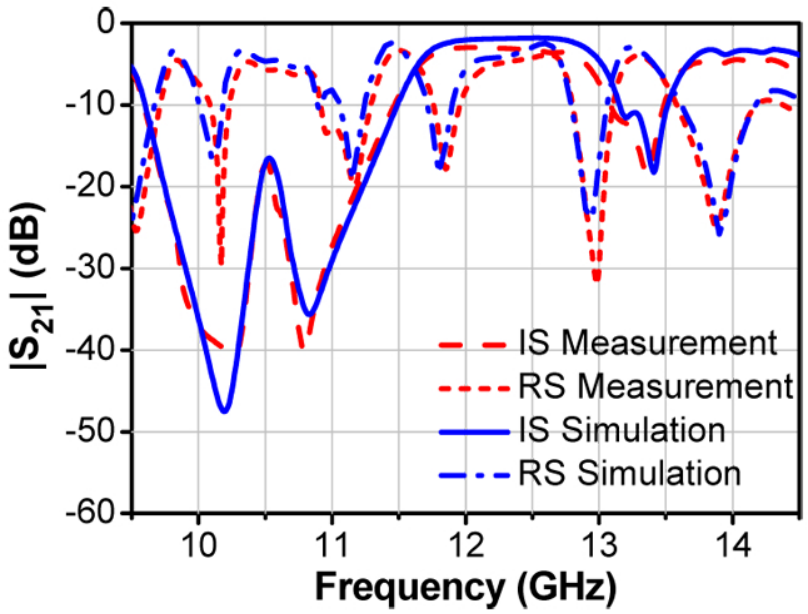

FIG. 7 Measured and simulated transmission coefficients of the final 3D realization of the proposed interdigital structure (IS) and the reference structure (RS).

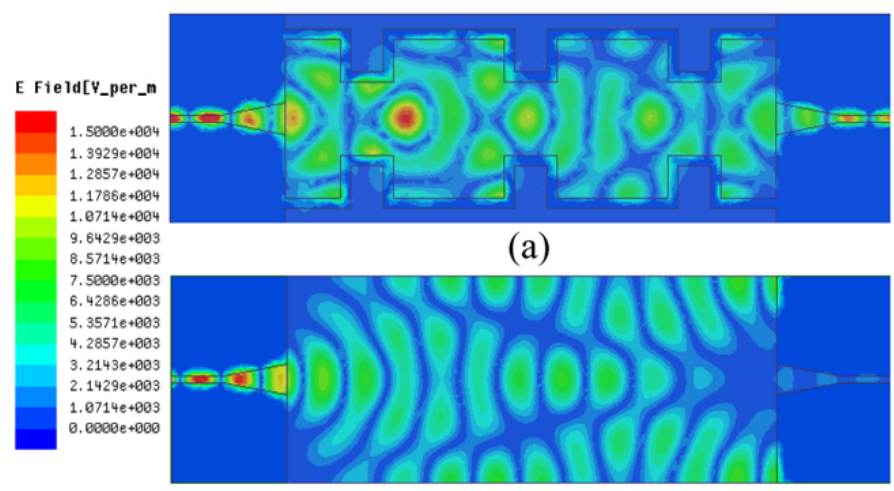

(b)

FIC. 8 Electric field distribution of the final 3D realization of (a) the proposed structure and (b) the reference structure at $13.8 \mathrm{GHz}$.

reference structure with above dimensions are simulated by the full-wave EM solver Ansoft High Frequency Structure Simulator (HFSS). And both structures are fabricated by Rogers RT/Duriod 5880 and shown in Figure 6. The simulated and measured transmission responses are shown in Figure 7, where good agreements between the simulations and measurements can be clearly seen.

For the final realization of the proposed structure in actual $\mathrm{PCB}$, the maximum transmission frequency in the interested frequency range is at $13.8 \mathrm{GHz}$ which is almost the same with 13.6 GHz in the $2 \mathrm{D}$ calculation, demonstrating the validity of the realization process. Although the power transmission in the final realization of the proposed structure at this frequency is $47.9 \%$ (the maximum transmission coefficient is $-3.2 \mathrm{~dB}$ ), much lower than $91.9 \%$ in the $2 \mathrm{D}$ case, the power transmission of the reference structure is only $0.7 \%$. Therefore, the influence of the proposed structure on the transmission response can still be demonstrated. The power transmission of the final realization of the proposed structure may be greatly improved by further tuning the length and width of the tapered transition line to excite the structure more efficiently, as well as decreasing the relative permittivity and thickness of the substrate to better approximate to the $2 \mathrm{D}$ case. 


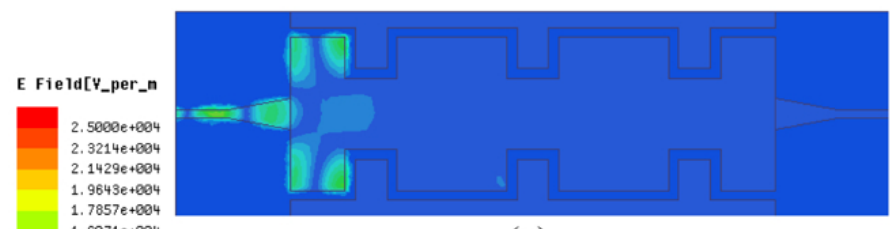

(a)

$4286 e+80$ $1.2500 e+284$ $1.0714 e+804$ $9.9286+203$ $5.3571 \mathrm{e}+800$ 3. $5714 \mathrm{e}+60$ $1.7857 e+803$ $0.8000++800$

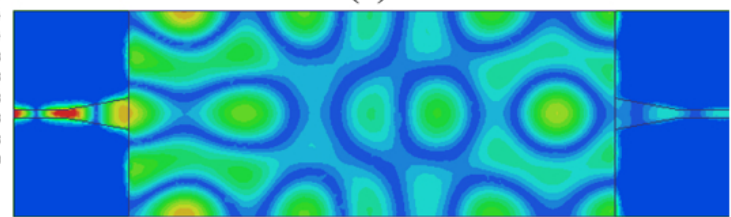

(b)

FIG. 9 Electric field distribution of the final 3D realization for (a) proposed structure and (b) the reference structure at $10 \mathrm{GHz}$.

As can be seen in Figure 7, there are an obvious bandgap from 9.7 $\mathrm{GHz}$ to $11.6 \mathrm{GHz}$ and a minor bandgap from $13.1 \mathrm{GHz}$ to $13.5 \mathrm{GHz}$ for the final realization of the proposed structure. The normalized frequencies of the bandgap $\omega a / 2 \pi c$ are from 1.00 to 1.20 and from 1.35 to 1.40 , which are just located within the bandgap obtained by the 2D calculation in Figure 4(b). This indicates the correctness of the final 3D realization of the new interdigital structure.

To explain the characteristics of the final realization, the Efield distribution at the maximum transmission frequency and the frequency within the bandgap are investigated. As for the E-field distribution at the maximum transmission frequency illustrated in Figure 8, the incident E-field propagates uniformly in the reference structure and little power is obtained at the received port; while the E-fields are constructively interfered in the final realization of the proposed structure and the received power is significantly improved. This phenomenon is very similar to the $2 \mathrm{D}$ calculation shown in Figure 3. As for the E-field distribution at $10 \mathrm{GHz}$ within the bandgap shown in Figure 9, little E-filed can enter the final realization of the proposed structure and a large amount of E-field still exists in the reference structure. The E-field distribution under both circumstances demonstrated above further testifies the validity of the implementation process of the new interdigital structure.

It should be noted that in the 2D calculation, the air medium around the metallic interdigital structure seems infinite. However, total width of the metal in the final 3D realization needs to be limited. Therefore, the final realization of the proposed structure shown in Figure 5(a) with different total widths is simulated to investigate how this parameter will affect the performance. Figure 10 shows the transmission coefficients corresponding to different total widths, where little influence of the total width on the transmission performance can be clearly seen. This will bring much convenience to the actual applications of the proposed structure.

\section{CONCLUSION AND FUTURE WORK}

A new design concept of periodic interdigital structure is presented from the perspective of EM field in this paper. Based

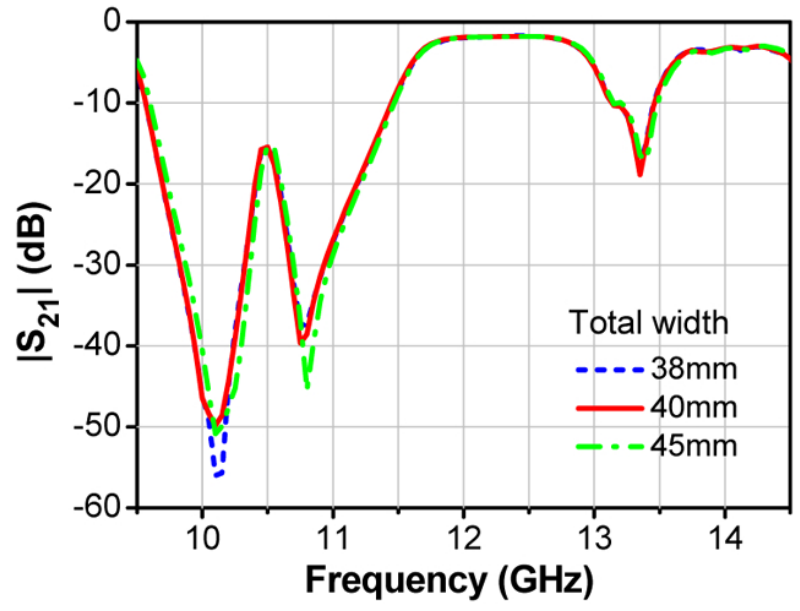

FIG. 10 Transmission coefficients of the final 3D realization for proposed structure with different total widths.

on 2D FDTD calculation of the proposed structure, the power transmission coefficient is obtained, demonstrating the nearly total transmission characteristic of the structure. And the interaction of EM fields with the structure is revealed to better understand the structure design. The band structure is also calculated to investigate the bandgap. For the final 3D realization in actual PCBs, the proposed interdigital structure is reversed from the viewpoint of energy confinement and an efficient excitation is obtained. The maximum transmission frequency, bandgap, and E-field distribution in the calculation of the final 3D realization are consistent with the 2D calculations, which all verify the implementation process of the proposed structure.

A direct application of the proposed structure is to construct a wideband bandstop filter by using the bandgap. With the help of other techniques (like the defected ground structure) to generate a new bandgap, a bandpass filter could be implemented by adjusting the nearly total transmission region between the bandgap of the proposed structure and the new bandgap. Moreover, we can let the reflected wave caused by the unmatched load pass through the two proposed structures with different phase delays. By adjusting the length of the two structures, we may make their outputs interfere destructively to realize the impedance matching. Theoretically, this method for impedance matching is applicable for any kind of loads. Our future work will be focused on the concrete applications of the new interdigital structure.

\section{ACKNOWLEDGEMENT}

This work is jointly funded by National Natural Science Foundation of China (NSFC) and Civil Aviation Administration of China (CAAC) (61079019), and supported by the Fundamental Research Funds for the Central Universities.

\section{References}

[1] J. D. Joannopoulos, S. G. Johnson, J. N. Winn, and R. D. Meade, Photonic Crystal: Modeling the Flow of Light (Princeton Univ. Press, Princeton, 2008). 
[2] R. Coccioli, F.-R. Yang, K.-P. Ma, and T. Itoh, "Aperture-coupled patch antenna on UC-PBG substrate," IEEE T. Microw. Theory 47, 2123-2130 (1999).

[3] F. Yang, and Y. Rahmat-Samii, “Microstrip antennas integrated with electromagnetic band-gap structures: A low mutual coupling design for array applications," IEEE T. Antenn. Propag. 51, 2936-2946 (2003).

[4] S.-G. Kim, H. Kim, H. Kang, and J.-G. Yook, "Signal integrity enhanced EBC structure with a ground reinforced trace," IEEE T. Electron. Pack. M. 33, 284-288 (2010).

[5] D. Nashaat, H. A. Elsadek, E. A. Abdallah, M. F. Iskander, and H. M. El Hennawy, "Ultrawide bandwidth $2 \times 2$ microstrip patch array antenna using electromagnetic band-gap structure (EBC)," IEEE T. Antenn. Propag. 59, 1528-1534 (2011).

[6] J.-W. Baik, S.-M. Han, C. Jeong, J. Jeong, and Y.-S. Kim, “Compact ultra-wideband bandpass filter with EBC structure," IEEE Microw. Wirel. Co. 18, 671-673 (2008).

[7] M. Al-Joumayly, and N. Behdad, "A new technique for design of low-profile, second-order, bandpass frequency selective surfaces," IEEE T. Antenn. Propag. 57, 452-459 (2009).

[8] M. Rotaru, and J. Sykulski, "Compact electromagnetic bandgap structures for notch band in ultra-wideband applications," Sensors 10, 9620-9629 (2010).
[9] B. Mohajer-Iravani, and 0. M. Ramahi, "Wideband circuit modal for planar EBC structures," IEEE T. Adv. Packaging 33, 169-179 (2010).

[10] M. Coulombe, S. F. Koodiani, and C. Caloz, “Compact elongated mushroom (EM)-EBG structure for enhancement of patch antenna array," IEEE T. Antenn. Propag. 58, 1076-1086 (2010).

[11] N. W. Ashcroft, and N. D. Mermin, Solid State Physics (Saunders College, Orlando, 1976).

[12] Rsoft Fullwave User Guide (RSoft Design Group, Ossning, 2003).

[13] C. Goffaux, J. Sánchez-Dehesa, and A. Levy Yeyati, "Evidence of fano-like interference phenomena in locally resonant materials," Phys. Rev. Lett. 88, 225502-1-225502-4 (2002).

[14] Y. S. E. Abdo, M. R. Chaharmir, J. Shaker, and Y. M. M. Antar, "Efficient excitation of an EBG guide designed using a defect triangular lattice of holes," IEEE Antenn. Wirel. Pr. 9, 167-170 (2010).

[15] Y. S. E. Abdo, M. R. Chaharmir, J. Shaker, and Y. M. M. Antar, "Investigation of the transition between two different EBG waveguides," IEEE Antenn. Wirel. Pr. 9, 1002-1005 (2010).

[16] D. M. Pozar, Microwave Engineering (Wiley, New York, 2005). 